\title{
Optical control system and optical actuator as its interface
}

\author{
TAKESHI NAKADA*, YASUSHI MORIKAWA* AND CAO DONG-HUI** \\ *Instrumentation and Control Division, Applied Physics and Information Science Department \\ Mechanical Engineering Laboratory, AIST, MITI \\ 1-2 Namiki,Tsukuba, Ibaraki, 305, JAPAN \\ **Hitachi Construction Machinery Co.,Ltd \\ 650 Kandatsu, Tsuchiura, Ibaraki, 305, JAPAN
}

\begin{abstract}
This paper deals with a new concept for constructing an optical control system with the minimum influence on operation environments and outlines the current status of the development of an optical actuator as an interface required to construct the optical control system. It describes that an optical actuator with the use of PLZT ceramics was found to be promising as a solid-state light-to-mechanic interface on the basis of the experimental results on step and on/off responses. To use the optical actuator practically, it is necessary to improve the response speed. The paper shows the possible way for the improvement.
\end{abstract}

\section{KEYWORDS}

Optical Control, Optical Actuator, Photo-pneumatic Interface, PLZT Ceramics, Photostrictive Effect

\section{INTRODUCTION}

Electric and fluid power control systems have played important rolls on the advances of mechatronics systems including robots and NC machine tools. Future mechatronics systems are desired to operate in cooperation with human beings. In addition, it is now actively required to develop mechatronics systems having the minimum influence on operation environments through their safety and reliability. Mechatronics systems operating in electromagnetic noise environments should rather be fluid power control systems. For signal transmissions within the systems, it would be indispensable to incorporate optical operations. On the basis of the recent status, opto-fluid control systems and light-to-mechanic (or -fluid) interface devices used in the systems have been studied and developed.

This paper proposes a new concept of optical control systems with the minimum influence on operation environments. The paper also introduces the current status of the development of optical actuators required for the system constructions and the operating performances of the bimorph type optical actuator with PLZT ceramics, which is now developed by the authors. The bimorph type optical actuator would have the adaptability to the applied field of piezoelectric actuators now used practically. The key for its practical use is to improve the response speed.

\section{CONCEPT OF OPTICAL CONTROL SYSTEM}

As the advance of the mechatronics technology, control systems having excellent features such as industrial robots have been developed. For their future advances, an important view is to enable them to operate safely in cooperation with human beings. For example, it has been reported that many 


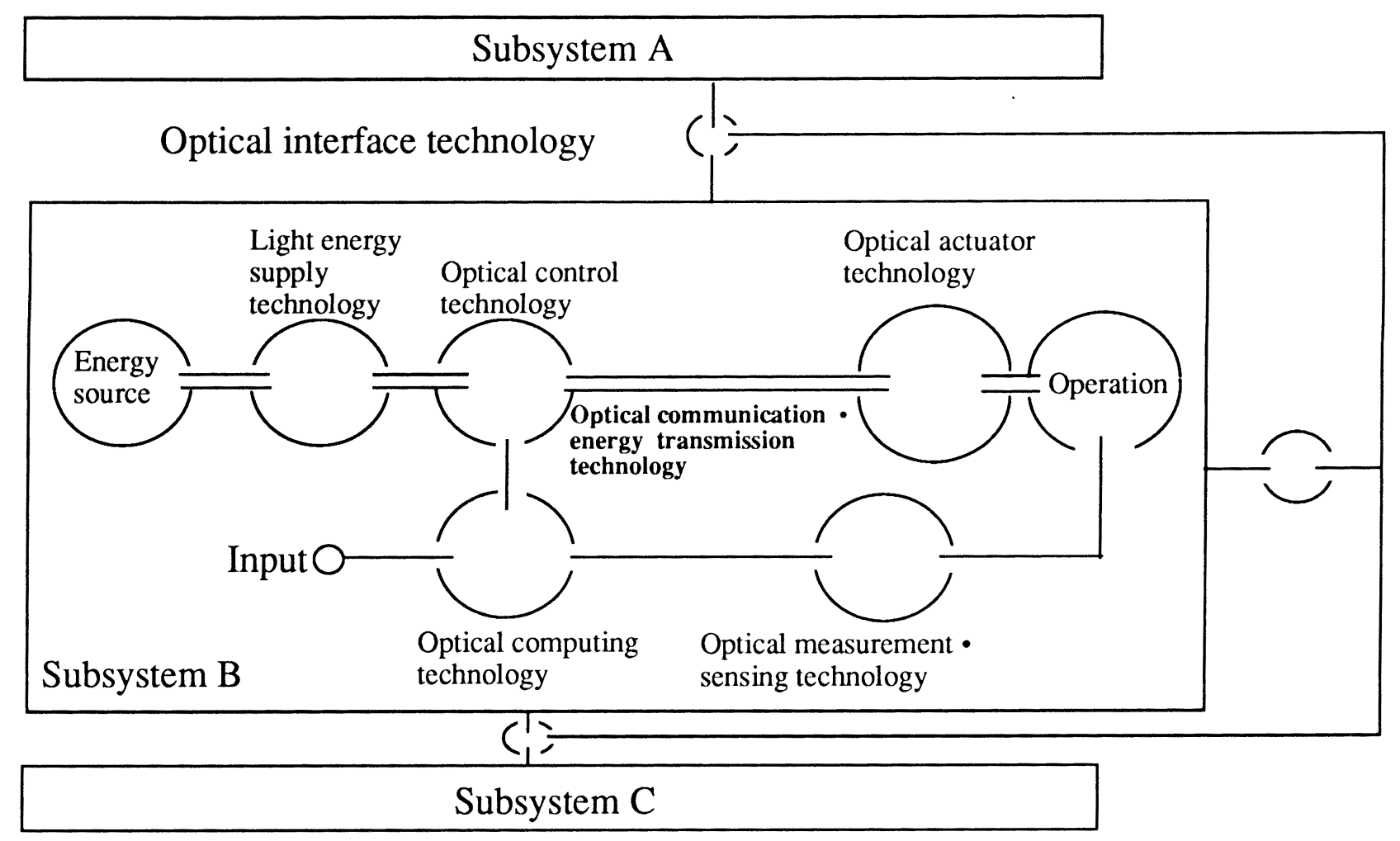

Figure 1. Concept of optical control system

malfunctions of a robot - an important factor of its safety and reliability - often appear due to some interference from electromagnetic noises generated by large-scale electric and/or mechatronics equipment installed around the robot. On flight control systems of airplanes, a variety of electronic units tend to be installed within them for the improvement of their light weight, economy, and maneuverability. An important problem for the flight control systems is the influences of electromagnetic interference generated in and out of the plane and lightning. Thus, it is strongly desired to realize control systems which can fully withstand electromagnetic noises for their safety and reliability. The current measure against electromagnetic noises is only dependent on a symptomatic way by shielding the noises. As an essential measure, optical devices should be installed which do not affected by electromagnetic noises. From the standpoint, the authors propose the idea of an optical power control system shown in Fig. 1. Different from conventional systems, the optical power control system in Fig. 1 is based on an entirely new idea, in which the signal level optical technologies of optical communication/transmission and optical measurement/sensing are integrated with a power level optical technology of optical actuator to control both optical information and power synthetically. Of course, the system partially includes electric and electronic technologies in the minimum level as necessity.

\section{OPTICAL ACTUATOR TECHNOLOGY}

The optical energy supply, optical communication/transmission, optical measurement/sensing technologies found in Fig. 1 have been explored substantially. A part of them are now used practically. On the optical computing technology, machines incorporating optical information processing theories are developed and optical operation architectures are studied. They are expected to be developed remarkably. On the other hand, the optical actuator technology dealing with optical power is now hardly studied and developed. The technology is a key to realize optical power control system.

Optical actuators have not been used practically. However, by the acess of converting optical inputs into mechanical outputs, optical actuators may possibly be realized in the three ways shown in Fig. 2. 

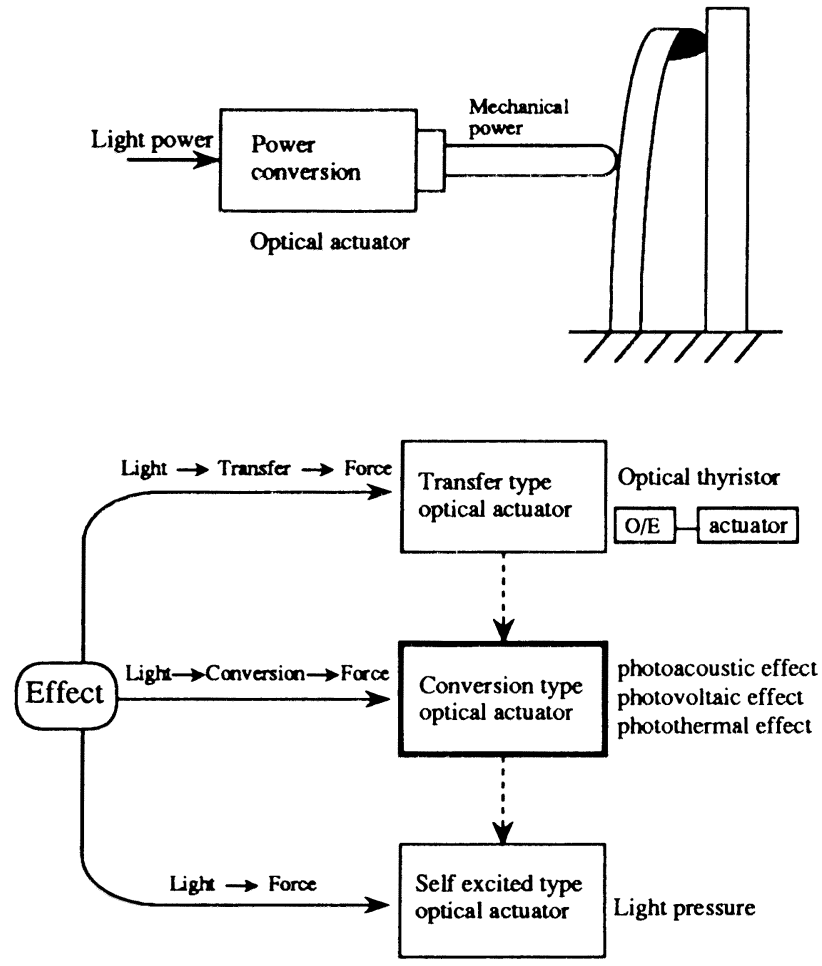

Figure 2. Clasificaltion of optical actuators

A transfer type optical actuator converts optical signals into electric signals by using photoelectric power transducers such as optical thyristor in order to drive a conventional electric actuator. It is feasible easily in the current technology level. However, the transfer type optical actuator cannot always be considered as an optical actuator with its advantages because photoelectric power transducers are required. A conversion type optical actuator converts optical power into another one internally to get final mechanical output. For the power transformation, it uses various physical or chemical phenomena including photo-acoustic effect, photostrictive effect, photo-thermal effect (such as thermal expansion or thermal modification), and photo-isomerization reaction. A self excited type optical actuator, which directly uses the pressure of light itself, is expected to have high speed response. However, it may be used in limited fields because its driving force is as small as about $10^{-9} \mathrm{~N}$. In the current state where the practical uses of optical actuators are put in the initial stage, the conversion type optical actuator is most promising. Photo-fluidic interfaces utilizing photoacoustic or photo-thermal effect and photopneumatic interface using laminar-to-turbulent transition of air jet (see Fig. 3) are now studied. In addition, solid-state optical actuators are also developed, which use shape memory alloys or photo-isomerization reaction materials (see Fig. 4 and 5).

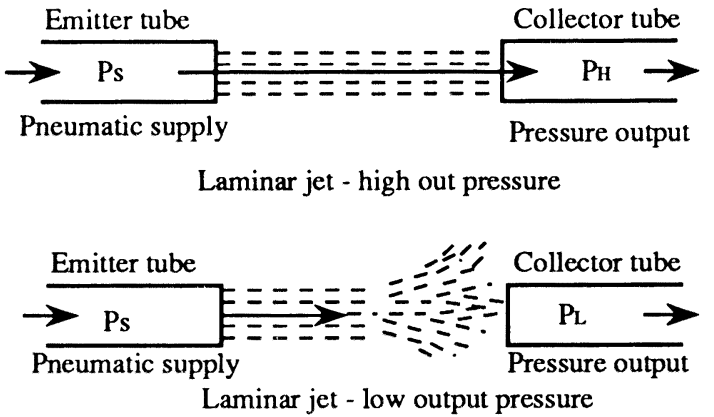

(a) The laminar-to-turbulent transition

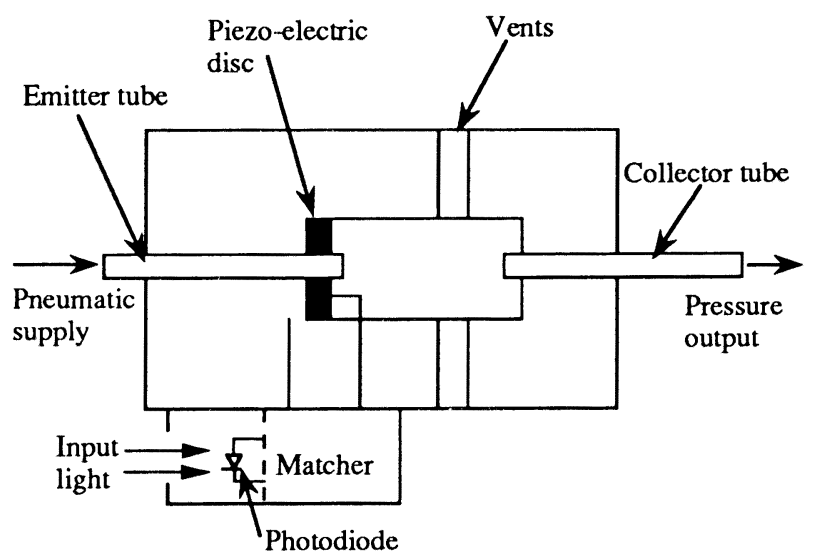

(b) Configuration of opto-pneumatic interface

Figure 3. Opto-pneumatic interface

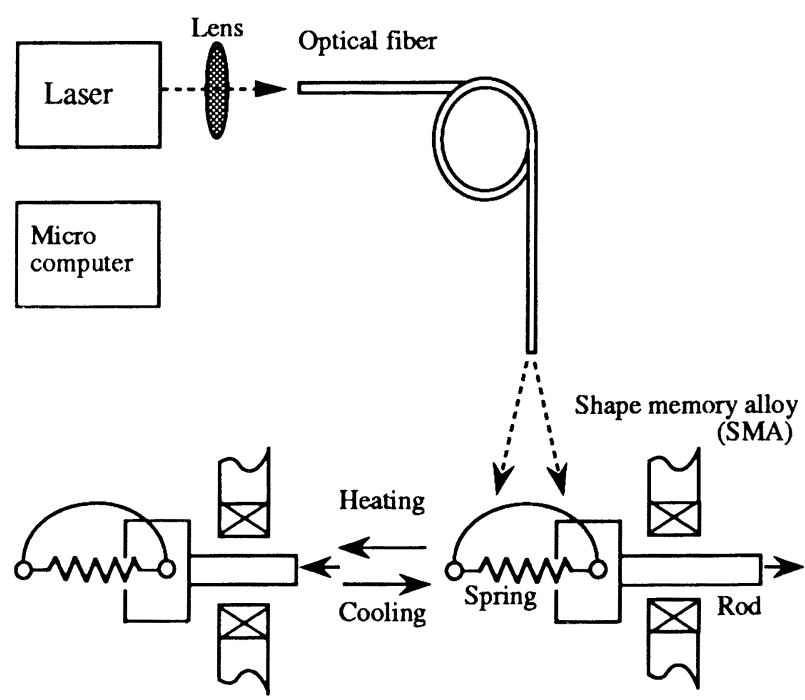

Figure 4. Optical actuator using SMA 


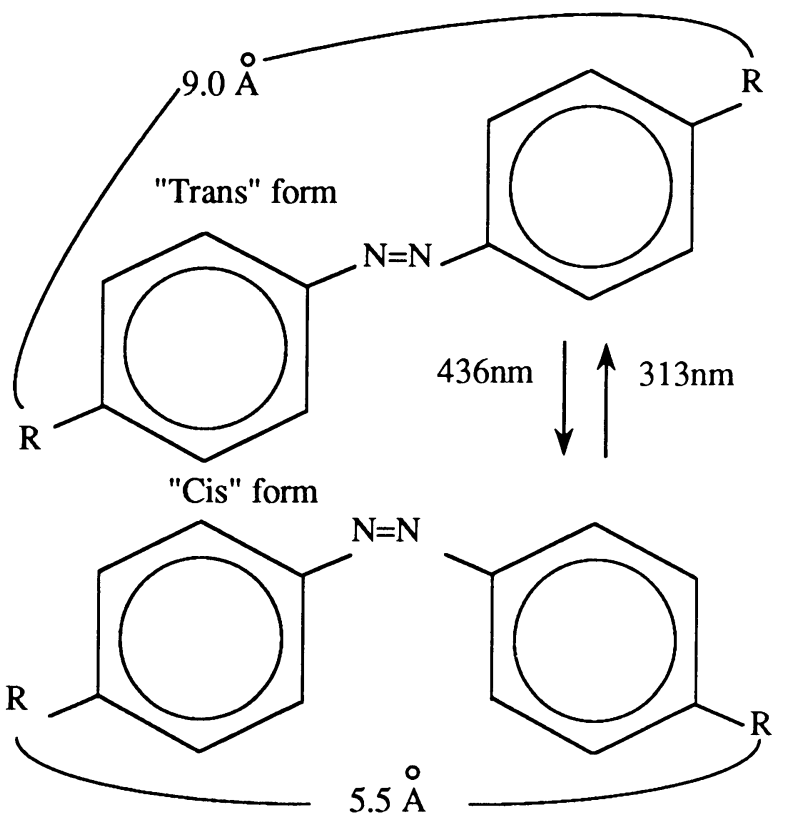

Figure 5. Photo-isomerization reaction in azobenzene
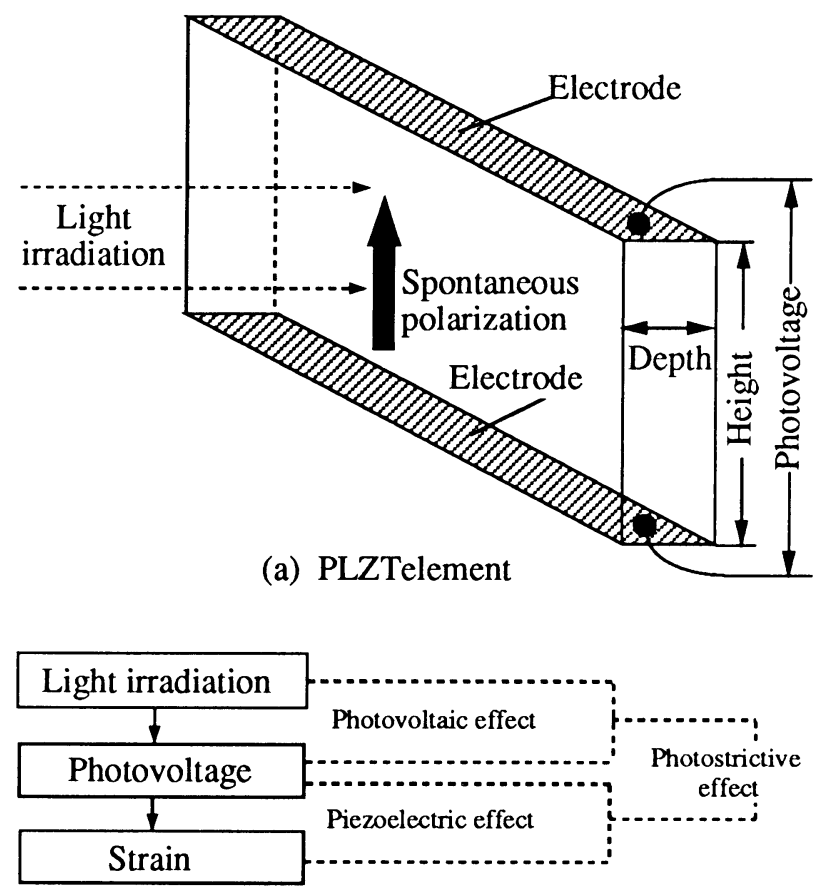

(b) Photostrictive effect

Figure 6. PLZT element and photostrictive effect

\section{BIMORPH TYPE OPTICAL ACTUATOR WITH PLZT CERAMICS}

The authors now develops the bimorph type optical actuator with PLZT ceramics. This section introduces the outline.
The PLZT ceramics is a type of piezoelectric materials of PZT ceramics to which La is added. If ultraviolet light having the wavelength of about 365 $\mathrm{nm}$ is irradiated on the PLZT element shown in Fig. 6(a), the photovoltaic effect causes high electro motive force to occur between the electrodes of the element. Because the PLZT element has the piezoelectric effect, the superposition with the photovoltaic effect generates some mechanical strain in the element. This series of effects is called the photostrictive effect (see Fig. 6(b)).

From fundamental experiments on the photostrictive effect of the PLZT element, we found that the PLZT element is subject to displacement by almost as large as the conventional PZT element but at slow response speed in seconds. Some experiments proved that the slow response speed is caused by the photovoltaic effect. Accordingly, we made detailed experiments on the photovoltaic effect. The experiments resulted in the lumped parameter model of the photovoltaic effect shown in Fig. 7. In Fig. 7, parameter $L$ is the intensity of the light radiated on the PLZT element; $C$ and $R$ are the electric capacity and resistance of PLZT element in the light radiation state, respectively; and $V_{p}$ is the photovoltage. As the result, we found that, if the time constant $T(=C R)$ of the step response of the device for $V_{p}$ is selected as an index of the response speed to the photovoltaic effect, the electric resistance $R$ is large in the T $\Omega$ order to slow the response speed. To improve the response speed, it is necessary not only to make the intensity of the radiation light higher and also to develop a new PLZT ceramic material having the features of larger photocurrent and smaller electric resistance and capacity.

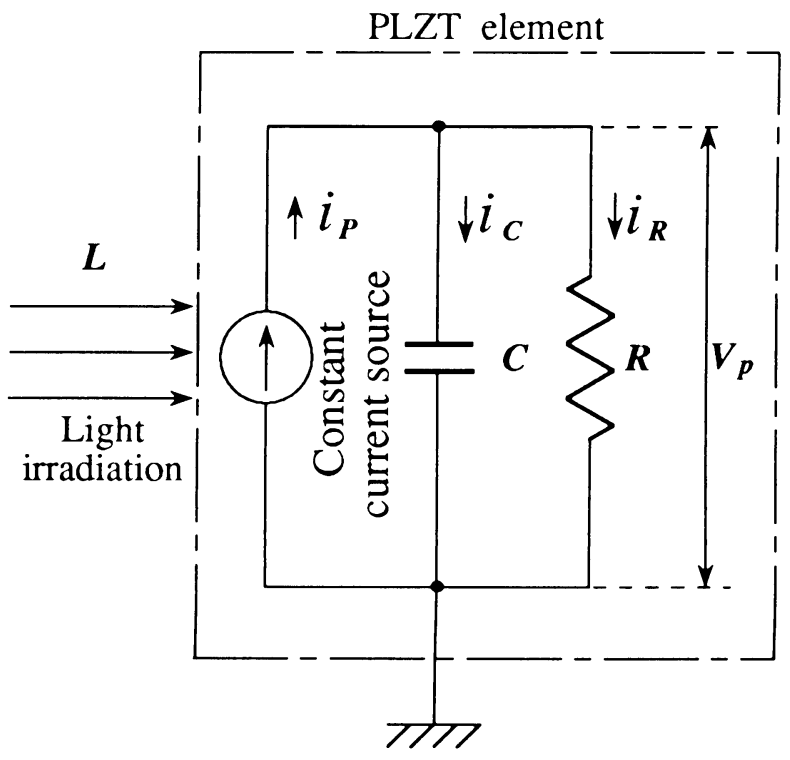

Figure 7. Electrical model for PLZT element 


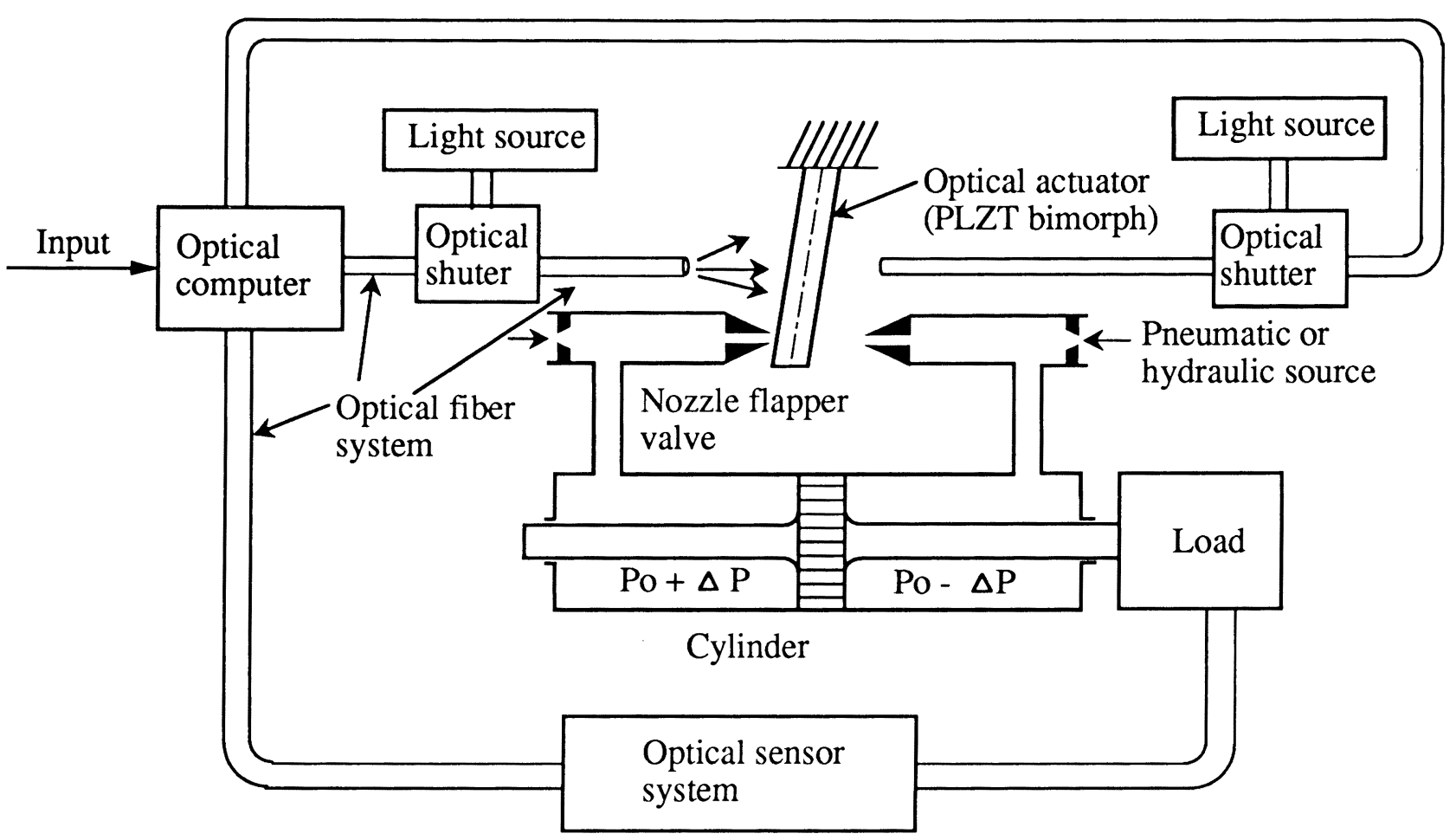

Figure 8. Photo-pneumatic control system

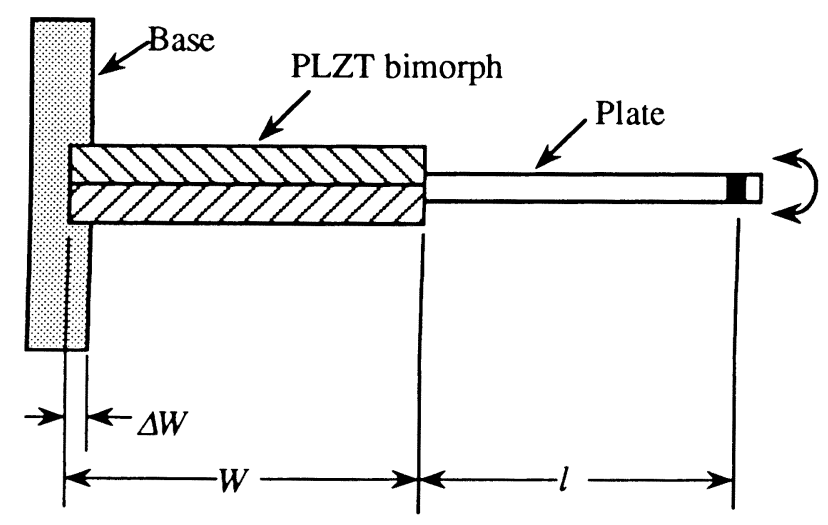

Figure 9. Structure of bimorph type optical actuator

For the final purpose of developing the optopneumatic control system shown in Fig. 8, we now advance the study of an optical actuator for controlling a nozzle flapper valve. As shown in Fig. 9 , the optical actuator has the bimorph structure with two PLZT elements of the same shape pasted with each other. The tip of the bimorph is bent upward if the upper surface of the bimorph is irradiated with light and downward if the lower surface of the bimorph is irradiated. Fig. 10 shows the step response of the displacement of the actuator tip occurring when either surface of the bimorph type actuator is irradiated with ultraviolet light of a step shape with optical intensity of $960 \mathrm{~W} / \mathrm{m}^{2}$. The optical actuator almost has the first order lag characteristic. Accordingly, we selected the final value $X_{e \infty}$ of the optical actuator displacement and the time constant $T_{e}$ corresponding to the response speed as the indexes presenting the performance of the optical actuator. Then we got $X_{e \infty}=1.4 \times 10^{-4} \mathrm{~m}$ and $T_{e}=18.0 \mathrm{sec}$.

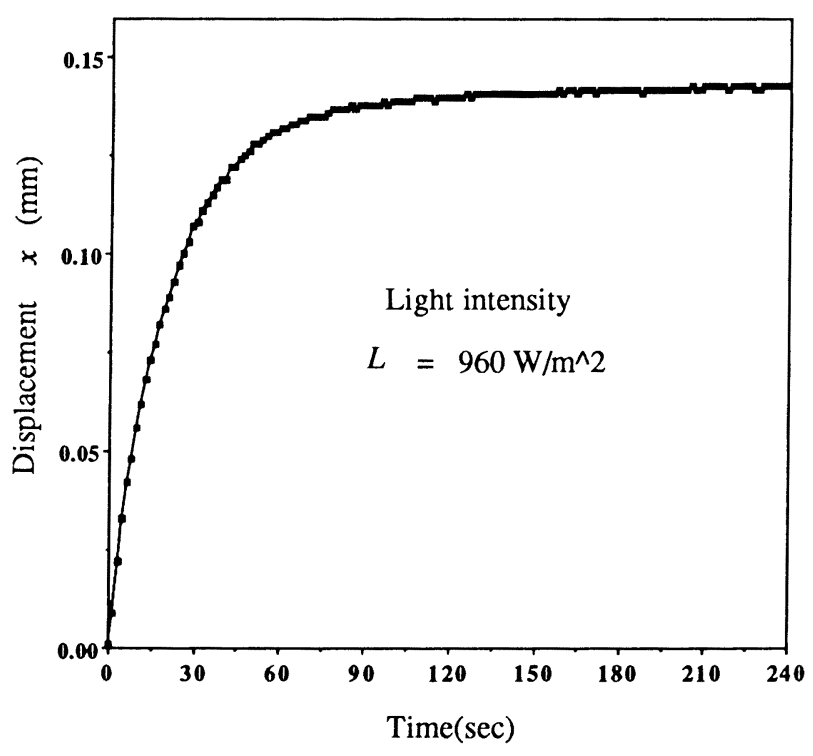

Figure 10. Step response of bimorph type optical actuator (Experiment) 
Fig. 11 shows an experimental result of the on/off driving response of the optical actuator occurring when the both surfaces of the optical actuator are alternately irradiated with ultraviolet light with the intensity of $960 \mathrm{~W} / \mathrm{m}^{2}$. The result indicates that the operation of the optical actuator follows the input almost immediately.

\section{CONCLUSIONS}

The optical actuator with PLZT ceramics has almost the same displacement performance as the PZT ceramics actuator. Thus, the improvement of the response speed would be the major key of its practical use. Higher intensity of the ultraviolet light and decreased in the thickness of the optical actuator as much as possible may be effective to improve the response speed. However, it is essentially desired to develop an entirely new material by injecting relevant elements into the base material. If such improvements enabled the optical actuator with PLZT ceramics to have a faster response, the optical actuator could be expected to have wide applications including micro-machine actuator, optical relay, and photophone as well as the opto-fluid power control system shown in Fig. 8. It is because the optical actuator has a variety of advantages such as no influence of electromagnetic interference and possible supply of non-contact energy.

The twenty first century is often called the era of light. In the century, optical control systems having the minimum influence on operation environments and excellent features hardly be realized by the conventional mechatronics systems may be constructed by actively advancing the developments of a variety of optical actuators in the initial stage currently including the PLZT ceramics.

\section{REFERENCES}

1. J.O.Gurney, Jr., Photofluidic Interface, Trans. ASME, J. of D. S. C., 1986, 160-3, 90-97.

2. S. Dohta, M. Tokai and T. Takamori, Studies on Photofludic Interface, Trans. SICE, 1990, 26-7, 780-786.

3. K. Yamamoto, Characteristics of an Opto-Fluidic Converter Utilizing Photoacoustic Effect, Trans. SICE, 1991, 27-12, 1405-1411.

4. H C Bramley M. A., Optical Control of a Pneumatic Actuator, Invited Lectures based on JHPS International Exchange Fund, 1992, 45-49.

5. T. Nakada et.al, Study on Optical ServoSystem (Contribution to Optical Actuator), Trans. JSME, 1991, 57-542, 116-121.

6. T. Nakada et.al, Study on Optical Servo System (Modelling for Photovoltaic Effect in PLZT

Element), Trans. JSME, 1992, 58-552, 189-194.

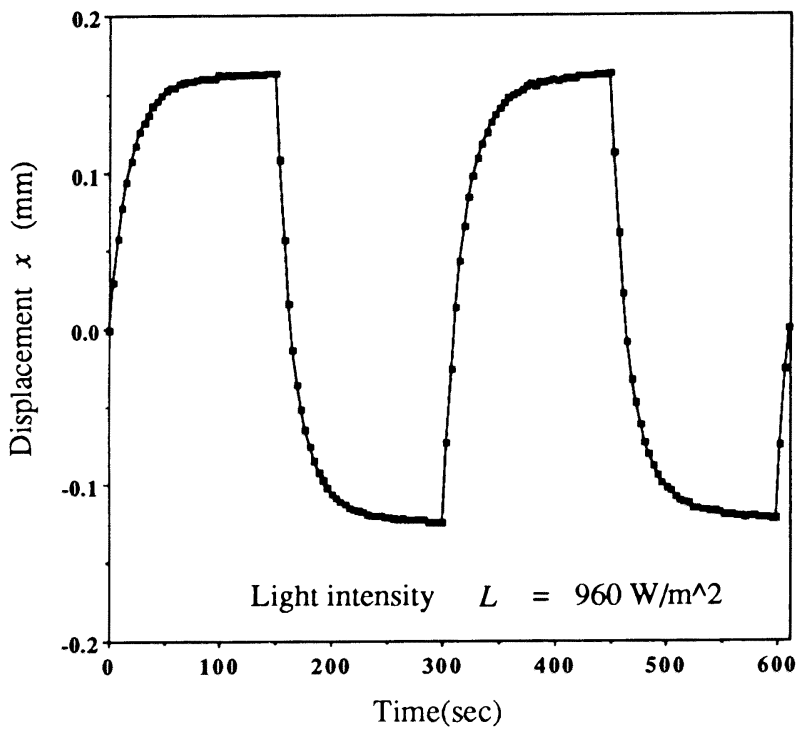

Figure 11. On-off response of bimorph type optical actuator (Experiment) 